\title{
THE EFFECTIVENESS OF SNAIL MUCUS (ACHATINA FULICA) AND CHITOSAN TOWARD LIMFOSIT PROLIFERATION IN VITRO
}

\author{
AGNES SRI HARTI ${ }^{1 *}$, ATIEK MURHARYATI ${ }^{1}$, DWI SULISETYAWATI ${ }^{1}$, MERI OKTARIANI ${ }^{1}$ \\ ${ }^{1}$ Departement of Nursing, Kusuma Husada Surakarta School of Health Science, Surakarta, Indonesia. \\ Email: agnessriharti@yahoo.com
}

Received: 15 September 2018, Revised and Accepted: 18 September 2018

\section{ABSTRACT}

Objective: The objective of this research was to assess the effectiveness of mucous and chitin slime cream against lymphocyte proliferation in vitro.

Methods: The research methods include snail mucus isolation, snail mucus cream material preparation, and lymphocyte cell proliferation test of 3-(4,5-Dimethylthiazol-2-yl)-2,5-diphenyltetrazolium bromide (MTT) reduction method.

Results: Lymphocyte cell proliferation test of MTT reduction method showed that chitosan $5 \%$ gave the most effective result toward lymphocyte proliferation activity compared to $100 \%$ snail slime and $5 \%$ snail slime cream. The $5 \%$ snail mucus cream $5 \%$ provides a higher proliferative activity than the $100 \%$ snail mucus. Positive control using Con A solution. Negative control was treatment without addition of test solution. The differences in lymphocyte proliferation activity were due to the presence of active compounds in chitosan, snail mucus, and snail slime cream that potentially increase lymphocyte proliferation. Significant differences in lymphocyte cell proliferation as Group K1, K2, K3, K4, K5, and K6.

Conclusion: The effectiveness of snail and chitosan slime cream toward lymphocyte proliferation by in vitro that chitosan $5 \%$ gave the most effective result toward lymphocyte proliferation activity compared to $100 \%$ snail slime and $5 \%$ snail slime cream.

Keywords: Anti-inflammatory, In vitro, Lymphocyte, Proliferation, Snail mucus, Wound healing, In vitro.

(C) 2018 The Authors. Published by Innovare Academic Sciences Pvt Ltd. This is an open access article under the CC BY license (http://creativecommons. org/licenses/by/4. 0/) DOI: http://dx.doi.org/10.22159/ajpcr.2018.v11s3.30041

\section{INTRODUCTION}

Inflammation is a complex biologic response of the vascular tissue to hazards such as pathogens, cellular damage, or irritation. Uncontrolled inflammation can also cause illness such as fever, atherosclerosis, and rheumatoid arthritis. Inflammation can be differentiated into acute and chronic inflammation. Wound healing is a process of normalizing skin and tissue integrity through various stages of acute inflammation [1].

The use of chemical compounds for wound healing or chemotherapy such as Povidone-iodine sometimes give toxic effects based on in vitro cell-level research, so that other medications are needed using natural ingredients as antimicrobial ingredients such as snail mucus cream and chitosan. Healing with snail mucus cream and chitosan can be an alternative because it is easy to use, good scattering, does not clog skin pores and antibacterial effects.

Snail mucus contains chemicals such as achatin isolates, heparan sulfate, and calcium. The content of achatin isolates is useful as antibacterial and analgesic, while calcium plays a role in hemostasis. The effects of snail mucus as an anti-inflammatory agent will accelerate the inflammatory phase so that the lymphocyte proliferation phase of wound healing is much faster. Snail mucus is multifunctional such as healing cuts or scratches, gingivitis, and skin care.

Chitosan is a chitin derived compound that can be biologically degraded, non-toxic, non-immunogenic, and biologically compatible with animal body tissues. Chitosan has been widely used in biomedical and pharmaceutical fields because of its biodegradable, biocompatible, and non-toxic [2].

The objectives of this study were to identify the effectiveness of snail mucus cream (A. fulica) and chitosan toward lymphocyte proliferation in vitro. This research is expected to obtain data and facts scientifically that were the potency and the effectiveness of snail mucus cream and chitosan as an effective anti-inflammatory agent of lymphocyte proliferation process which has an important role in wound healing process.

\section{MATERIALS AND METHODS}

Materials

Chitosan (Biotech Surindo, Indonesia), snail mucus, bovine serum, Roswell Park Memorial Institute (RPMI)-1640 (fetal bovine serum [FBS], Glutamine, Penicillin, and Streptomycin), $\mathrm{HCl} 2 \mathrm{M}$, Aquadest, $\mathrm{NH} 4 \mathrm{Cl}, 3$ - (4,5-dimethyl-2-thiazolyl) -2, 5-diphenyl-2H-tetrazolium bromide (MTT) (E. Merck), methanol, isopropanol, Giemsa 20\%, Mice Balb/c, ELISA reagent kit for IgM and IgG (Sigma), medium RPMI 1640 (Sigma), penicillin, streptomycin, Fungizon, and Aquadest were used.

\section{Isolation of Snail Slime and Process Snail Slime Cream}

The sample of mucus snail (Achatina fulica) as in Fig. 1 was obtained from local snails. There are 10-50 snails were isolated by electric shocks of 5-10 volts, in 30-60 s. Mucus was macerated in water for $24 \mathrm{~h}$ at $40^{\circ} \mathrm{C}$. The water-soluble slime fraction was obtained from the procedure of mixing water twice of sample added to the mucus. The supernatant obtained was called WSF (Water-Soluble Fraction). The mucin fraction of WSF was obtained using precipitation ethanol which mixed the supernatant of water maceration with absolute ethanol with a ratio of $1: 3$, which was a common isolation method of mucus. WSF and the mixture of disentifuse were $2900 \mathrm{~g}$ for $30 \mathrm{~min}$. The obtained precipitation was dissolved with Tris $-\mathrm{Cl}$ and the Mucin fraction was obtained [3].

The serving of $5 \%$ cream preparations was obtained with formula stearic acid, Cera alba, Vaseline alba, nipagin, triethanolamine, propylene glycol, nipazole, Aquadest, and snail mucus including melting and emulsification processes. 
Table 1: Value of OD effectiveness of snail mucus cream and chitosan toward lymphocyte cell proliferation by in vitro Balb/c method MTT reduction

\begin{tabular}{lllllll}
\hline Plate & OD & \multicolumn{5}{l}{} \\
\cline { 2 - 6 } & Chitosan (K1) & Snail mucus (K2) & Snail mucus cream (K3) & Media (K4) & Positive control (K5) & Negative control (K6) \\
\hline A & 0.311 & 0.111 & 0.118 & 0.036 & 0.212 & 0.117 \\
B & 0.309 & 0.104 & 0.118 & 0.035 & 0.309 & 0.116 \\
C & 0.293 & 0.103 & 0.110 & 0.035 & 0.368 & 0.151 \\
D & 0.300 & 0.155 & 0.113 & 0.036 & 0.476 & 0.152 \\
E & 0.305 & 0.158 & 0.114 & 0.035 & 0.491 & 0.171 \\
F & 0.283 & 0.110 & 0.111 & 0.037 & 0.348 & 0.182 \\
G & 0.291 & 0.111 & 0.111 & 0.037 & 0.377 & 0.116 \\
H & 0.327 & 0.111 & 0.111 & 0.370 & \\
\hline
\end{tabular}

OD: Optical density, MTT: 3-(4,5-Dimethylthiazol-2-yl)-2,5-diphenyltetrazolium bromide
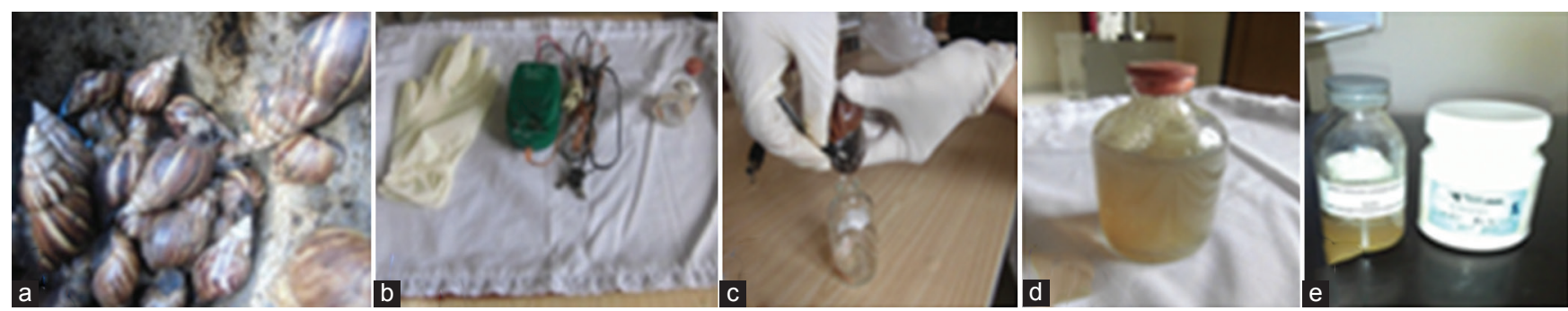

Fig. 1: Proses isolation and collection of snail mucus, (a) colecction sample, (b) equipment isolation, (c) collection of snail slime, (d) snail mucus, and (e) Snail mucus cream 5\%

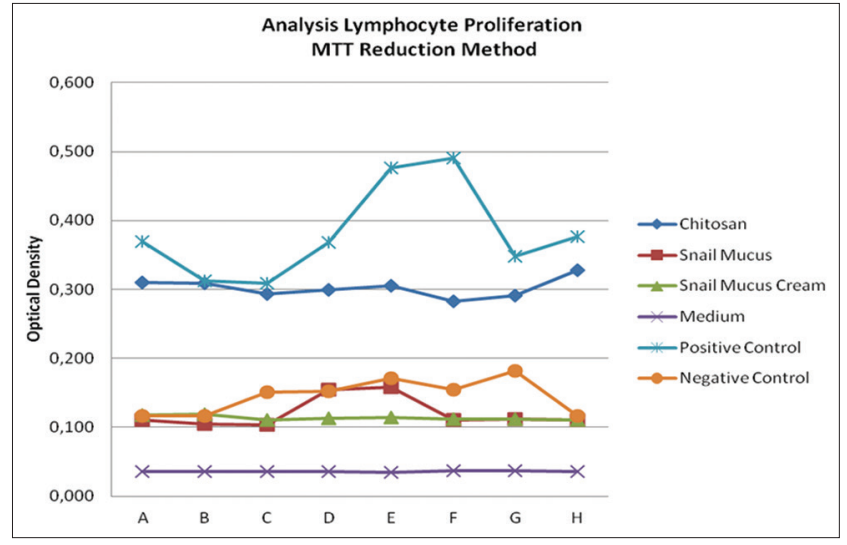

Fig. 2: Graph lymphocyte proliferation 3-(4,5-Dimethylthiazol-2yl)-2,5-diphenyltetrazolium bromide reduction method

The nonsoluble water components such as oil and wax were melted together in a water plate at $70-75^{\circ} \mathrm{C}$, while aqueous solutions were heat resistant; the water-soluble component was heated at the same temperature as the fat component. Then, the aqueous solution was slowly added to the liquid fat mixture and stirred constantly, the temperature being maintained for 5-10 min to prevent crystallization from wax or fat. The mixture was slowly cooled by continuous stirring, until the mixture is thickened. If the aqueous solution was not equal to the temperature of the fused fat, then some wax will become solid, resulting in the separation between the fat phase and the liquid phase. Cream preparations packaged such as ointment preparations in bottles or tubes.

\section{Lymphocyte culture}

The lymphocyte cell was isolated from the spleen of Balb/c mice aged 6-8 weeks aseptically. Spleen cells were taken by chopping one end of the spleen and bringing RPMI medium (Gibco, BRL) to the other end of the spleen placed on a Petri dish containing RPMI medium. The cells obtained were collected in a tube and centrifuged $2000 \times \mathrm{g}$ for $5 \mathrm{~min}$ at room temperature. The formed pellet was suspended with a $0.17 \mathrm{M} \mathrm{NH} 4 \mathrm{Cl}$ solution of $5 \mathrm{ml} / \mathrm{l}$ of spleen, to lyses red blood cells for $5 \mathrm{~min}$, at room temperature. Next centrifuged $2000 \times \mathrm{g}, 5 \mathrm{~min}$, at room temperature, then $\mathrm{NH} 4 \mathrm{Cl}$ solution and Lysates of red blood cells were discarded. The pellet of lymphocyte cells was washed with RPMI medium twice by centrifugation $2000 \times \mathrm{g}, 5 \mathrm{~min}$, at room temperature. The washed lymphocytes were resuspension with a growing medium of $1 \mathrm{~mL}$ RPMI containing 10\% fetal bovine serum, $100 \mathrm{IU} / \mathrm{ml}$ antibiotic penicillin, $100 \mu \mathrm{g} / \mathrm{ml}$ streptomycin, and $2 \mathrm{mM}$ L-glutamine. Columns for filtering $\mathrm{T}$ lymphocytes are prepared and saturated with RPMI medium and allowed to medium to wool nylon. The lymphocyte suspension was inserted into a column containing nylon wool and incubated for $30 \mathrm{~min}$. After $30 \mathrm{~min}$, the suspension was transferred into a sterile tube by slowly pressing the syringe of the syringe; this procedure was aimed at separating lymphocytes from B lymphocytes. In T lymphocyte suspension added RPMI $3 \mathrm{~mL}$ and centrifuged $2500 \mathrm{rpm}$ for $5 \mathrm{~min}$. The precipitate was taken and added $1 \mathrm{~mL}$ RPMI, then counted the number of cells using hemacytometer until the cell obtained with a density of $1 \times 106$ cells [4].

\section{Treatment stage}

The experimented animals (mice) were wounded by incising them with the measure of $0.5 \mathrm{~cm} \times 0.5 \mathrm{~cm}$ and depth of $0.3 \mathrm{~cm}$ on the back using a sterile scalpel. The treatment of research involved:

\section{K1: Chitosan $2 \%$ group}

K2: Snail mucus group

K3: Snail mucus cream $5 \%$ group

K4: Media control group

K5: Positive control group

K6: Negative control group.

\section{Lymphocyte cell proliferation MTT method}

Each of the $100 \mu \mathrm{L}$ suspension cells of lymphocytes with a density of $1.5 \times 106 / \mathrm{mL}$ in complete RPMI medium (RPMI+10\%, FBS+penicillin+streptomycin+Fungizon), were fed into microplate 96 wells with treatment and then cell cultures incubated at $37^{\circ} \mathrm{C}$ in the incubator $\mathrm{CO}_{2} 5 \%$ for 3 days $4 \mathrm{~h}$ before $72 \mathrm{~h}$ incubation, microculture plate removed from incubator $\mathrm{CO}_{2}$. Each well was given $20 \mu \mathrm{l}(100 \mu \mathrm{g})$ of MTT solution, and the cell culture was returned to the incubator. A total of $200 \mu \mathrm{l}$ of the medium from each well at the end of the 72-h incubation 

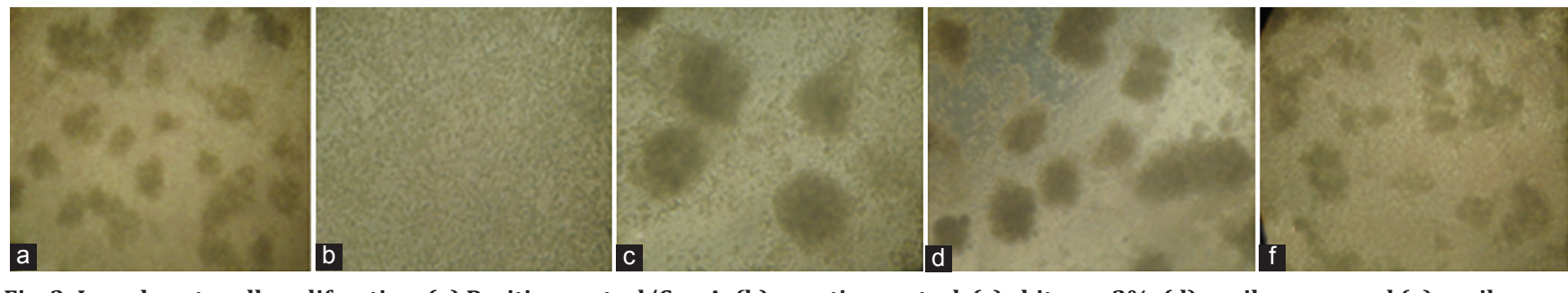

Fig. 3: Lymphocyte cell proliferation, (a) Positive control/Con A, (b) negative control, (c) chitosan 2\%, (d) snail mucus, and (e) snail mucus cream $5 \%$

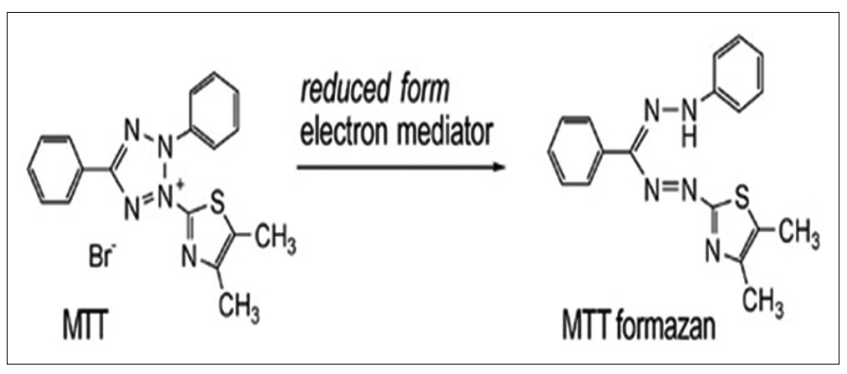

Fig. 4: Reduction reaction of 3-(4,5-Dimethylthiazol-2-yl)-2,5diphenyltetrazolium bromide

period was carefully aspirated using a micropipette. Furthermore, each well was added $100 \mu \mathrm{l}$ of isopropanol containing $0.04 \mathrm{~N} \mathrm{HCl}$ to resuscitate the formed Tetrazolium crystals. After the crystals dissolve, a purple solution is formed with intensity proportional to the rate of cell proliferation. The reaction was stopped with a stopper reagent, a solution of $10 \%$ SDS in $0.01 \mathrm{~N}$ hydrochloric acid of $100 \mu \mathrm{L} /$ well. Next read his optical density (OD) with an ELISA Photo Reader at a wavelength of $550 \mathrm{~nm}$. Cells in certain conditions are allowed not reconstituted to be photographed under of microscope [5] as in Fig.3.

\section{RESULTS AND DISCUSSION}

The effectiveness of snail mucus creams and chitosan toward lymphocyte proliferation in vitro

Based on the OD value data in Table 1 and as in Fig.2, lymphocyte cell proliferation test of MTT reduction method showed that chitosan $5 \%$ gave the most effective result toward lymphocyte proliferation activity compared to $100 \%$ snail slime and 5\% snail slime cream. The 5\% snail mucus cream $5 \%$ provides a higher proliferative activity than the $100 \%$ snail mucus. Positive control using Con A solution. Negative control was treatment without the addition of test solution. The differences in lymphocyte proliferation activity were due to the presence of active compounds in chitosan, snail mucus, and snail slime cream that potentially increase lymphocyte proliferation. Significant differences in lymphocyte cell proliferation as group K1, K2, K3, K4, K5, and K6.

The observation of immunostimulatory activity toward lymphocyte cell proliferation was done for $48 \mathrm{~h}$. This was related to the nutrients that become the lymphocyte cell intake during the culturing process. RPMI 1640 was a medium for cultured lymphocyte cells by in vitro that rich of organic salts, amino acids, vitamins, and glucose [6]. Glucose played an important role in providing energy for the work of lymphocyte cells. The limited use of glucose in lymphocyte cells for more than $48 \mathrm{~h}$ results in lymphocyte cells unable to proliferate well, which had a major impact on the survival of lymphocyte cells. While the observation of immunostimulatory activity is done more than $48 \mathrm{~h}$, then many lymphocyte cells were dead or did not maximize in proliferation [7].

Inflammation was a complex biological response of the vascular tissue in the presence of hazards, such as pathogens, cellular damage, or irritation. It was a self-protection effort of the organism to eliminate the injury to the wound and initiate the tissue healing process. Lymphocytes provided protection for the body against infection. B lymphocytes can proliferate and differentiate into plasma cells that secrete antibodies. $\mathrm{T}$ lymphocytes secrete lymphokines which were needed for the activation of lymphocytes and macrophages. Lymphocytes re-circulate from one lymphoid organ to the lymphatic tract and blood, so during infection, there would be many lymphocytes exposed to infectious germ antigens. The ability to recognize the antigen was due to the presence of receptors on the lymphocyte cell surface. Lymphocytes that have been stimulated by specific antigens will split and express new receptors to respond of cytokines from other cells which were the signals of proliferation. Lymphocytes will also secrete their own cytokines and under the influence of those cytokines will undergo a number of cleavage cycles before differentiating into mature effectors cells.

Uncontrolledinflammationcauseddiseases suchasfever, atherosclerosis, and rheumatoid arthritis. Inflammation can be differentiated into acute and chronic inflammation. Immunomodulator was a compound which affects the humoral and cellular immune system. Chitosan was a chitin derived compound that can be biologically degraded, non-toxic, nonimmunogenic, and biologically compatible with animal tissue [8]. Chitosan has a degree of deacetylation of $80-90 \%$, but in general, chitosan was expressed when its deacetylation degree was $>70 \%$. Chitosan was not soluble in water but acid solvents with a $\mathrm{pH}$ below 6.0. The common solvent used to dissolve chitosan is $1 \%$ acetic acid at a pH of about 4.0. At $\mathrm{pH}$ above 7.0, the solubility stability of chitosan was limited. At high $\mathrm{pH}$, chitosan forms a polyelectrolyte complex with anionic hydrocolloids producing gel. Chitosan had a hydroxyl and amine group that provide an intermolecular or intramolecular hydrogen link to make a strong hydrogen network, make the chitosan insoluble in water. The functional groups of chitosan (primary hydroxyl groups in C-6, secondary hydroxyl groups in C-3 and amino groups in C-2) cause the chitosan to be chemically modified. Chitosan had been widely used in biomedical and pharmaceutical fields because it was biodegradable, biocompatible, and non-toxic [9].

The potential of snail mucus in wound healing process was due to its active substance content. Snail mucus contained chemicals such as achatin isolates, heparan sulfate, and calcium. The achatin isolates were beneficial as antibacterial and analgesic, while calcium played a role in hemostasis. The effects of snail mucus as antibacterial and anti-inflammatory accelerated the inflammatory phase either the proliferation phase of wound healing. The content of snail mucus that affected the proliferation of fibroblasts was heparan sulfate which was useful in accelerating the wound healing process by helping the process of blood clotting and proliferation of fibroblast cells. Heparan sulfate also served for angiogenesis, inhibition of vascular endothelial growth factor or decreases the mitogenic activity of fibroblast growth factor (FGF). Heparan sulfate as one of the proteoglycans serves as a binder and reservoir for basic fibroblast growth factor (BFGF) which was secreted into extracelluler musculer (ECM). ECM can release BFGF which stimulated recruitment of inflammatory cells, activation of fibroblasts, and the formation of new blood vessels of each injury [10]. The addition of heparan sulfate concentration absorbed by tissue increased the proliferation of fibroblasts. Wound scrated which snail mucus stimulated tends for the proliferation of fibroblasts so that the amount of fibroblasts in the wound area was much and the wound will quickly closed [11]. 
Snail mucus (A. fulica) was able to heal wounds faster than the wounds given normal saline solution. Snail mucus was believed to contained certain nutrients that treated the wounds. The important content contained in snail mucus was glycosaminoglycan which binds copper peptide compounds [12]. The chemical composition of the snail was rich in protein. Achatin proteins in snails (A. fulica) had important biologic functions, such as bacterial protein binding (enzyme) receptors [13]. The use of snail mucus (A. fulica) with Chitosan ratio (1:2) was effective for wound healing within 5 days. The Biopreparation of snail mucus (A. fulica) was effective and can be applied to the membrane of chitosan as bandage wound for wound healing [14].

Wound healing was essential to restore skin integrity as soon as possible, and it was a complex and dynamic process. The proliferative phase involving fibroblast cells was an important step in wound healing. Drugs used to accelerate the inflammatory phase would accelerate the proliferation phase. The main role in this repair process was fibroblasts. Fibroblasts played a role in the production of protein structures used during tissue reconstruction. In particular, fibroblasts were the basic ingredients of collagen fibers which linked the wound edges. Wound healing was a process of normalizing skin and tissue integrity under it through various stages of acute inflammation. Healing was related to inflammation. Inflammation was an early process of wound healing. Before the process of healing, products from inflammation such as exudates and dead cells had moved from the region accompanied by melting of dead tissue. This event occurs due to the autolithic enzyme of the dead tissue itself or autolysis and the enzyme sent from inflammatory leukocytes. The liquid material was readily absorbed into the lymph vessels and paves the way for wound healing. Repairing the network included two concrete processes: Repair with regeneration and replacement with connective tissue (fibroplasias). Repaired with regeneration was indicated by the replacement of damaged cells and tissues with new ones. The improvement of connective tissue occurs through four stages: Migration and proliferation of fibroblasts, extracellular decomposition of the matrix, formation of new blood vessels (angiogenesis), and scar tissue maturation. Fibroblasts proliferate binding elements of ECM formed scar tissue and accelerate wound healing. Activated fibroblasts secrete the ECM, binding the element of the ECM into granulation tissue. The formation of granulation tissue ends the proliferation phase of the wound healing process and begins to maturation in the remodeling phase. The drugs used to accelerate the inflammatory phase accelerated the proliferation phase. Some factors would be affecting the wound healing process include immunological status; blood sugar levels impaired white cell function, hydration (slows metabolism); nutrition; blood albumin levels ("building blocks" for repair and colloid osmotic pressure edema); oxygen supply and vascularization; pain causes vasoconstriction, and corticosteroids depress immune function [17].

MTT assay was a more practical, fast, and efficient quantitative cell viability test method with a fairly accurate result compared to Trypan blue staining method performed manually on fungi cell testing. The principle of MTT dyeing method was by reducing the yellow Monotetrazolium salt and dissolve in water by the succinic enzyme dehydrogenase produced by living cells into a water-insoluble formazan blue crystalline to be the final product and then measured the blue density formed by the microplate reader. The breakdown of MTT became formazan carried out by the enzyme succinic dehydrogenize in the living cell mitochondria. The resulting absorbance was directly proportional to the concentration of formazan crystals formed. The absorbance value had a positive correlation to the number of living cells. Reduction of the tetrazolium salt was a reliable way to determine the proliferation of lymphocyte cells. The change in MTT, originally yellow to dark blue, indicated the presence of living cells that release the enzyme succinic dehydrogenize and reduce the dye of the MTT. In dying cell whose entire metabolism process stop and cannot produce an enzyme that can reduce MTT, it did not produce an end product formazan so as not to change the yellow color of the tetrazolium salt.
The color change that occurred then measured the absorbance using a microplate reader tool at a wavelength of $570 \mathrm{~nm}$. The reduction reaction of MTT was dead by the enzyme succinic dehydrogenize produced by living cells can be seen in Fig. 4 .

\section{CONCLUSION}

There was chitosan $5 \%$ gave the most effective result toward lymphocyte proliferation activity by in vitro compared to $100 \%$ snail slime and $5 \%$ snail slime cream. The innovation of snail mucus and chitosan $5 \%$ can be applied as galenic preparations of anti-inflammatory creams. Snail mucus creams and chitosan galenic preparations toward lymphocyte proliferation by in vitro were effective for wound healing.

\section{CONFLICTS OF INTEREST}

All authors have none to declare.

\section{REFERENCES}

1. Robbins C. Textbook of Pathology. Issue. 7, Vol. 1. Jakarta: Book Medical Publishers EGC; 2007.

2. Harti AS, Haryati DS, Setyaningsih WS, Yatmihatun S. The potential Chito-Oligo-saccharide (COS) as natural prebiotic and preservatives on synbiotic tofu in Indonesia. Int J Pharm Med Biol Sci 2015;4:204-8

3. Berniyanti T, Suwarno B. Characterization of snail slime protein (Achasin) isolate local as antibacterial factor. $\mathrm{J}$ Vet Media 2007;23:139-44.

4. Depamede S, Rosyidi A. Inhibition of Balb/c lymphocyte's proliferation inhibition by balinese cyst testis extract: The role of TGF- $\beta$. Livestock Media 2009;2009:95-103.

5. Mossmann, T. Rapid colorimetric assay for cellular growth and survival: Application to proliferation and cytotoxicity assays. J Immunol Methods 1983;65:55-63.

6. Yang Z, Xiong HR. Culture conditions and types of growth media for mammalian cells. In: Nelli LC, Matteoli E, editors. Biomedical Tissue Culture. Croatia: Intech Publisher, Open Acsess Company; 2012. p. 11.

7. Maciver NJ, Jacobs SR, Wieman HL, Wofford JA, Coloff JL, Rathmell JC, et al. Glucose metabolism in lymphocytes is a regulated process with significant effects on immune cell function and survival. J Leukoc Biol 2008;84:949-57.

8. Harti AS, Nurhidayati A, Handayani D, Kusumawati HN, Haryati DS. The fortification tempeh of rice bran chitosan as functional food antihypercholesterolemia in Indonesia. Int $\mathrm{J}$ Biosci Biochem Bioinform 2014:4:423-7.

9. Harti AS, Nurhidayati A, Handayani D. The potential of rice bran and chito oligosaccharide as natural prebiotic on traditional tempe in Indonesia. Int J Biosci Biochem Bioinform 2013;3:654-6.

10. Vieira TC, Costa-Filho A, Salgado NC, Allodi S, Valente AP, Nasciutti LE, et al. Acharan sulfate, the new glycosaminoglycan from Achatina fulica Bowdich 1822. Structural heterogeneity, metabolic labeling and localization in the body, mucus and the organic shell matrix. Eur J Biochem 2004;271:845-54.

11. Perez WP, Dina F, Iwang Y. Effect of mucus snail (Achatina fulica) against total cells fibroblasts on incision wound healing skin of mice (Mus musculus). J Vet Med Sci Health 2012;4:195-203.

12. Ali GP. Rapid Comparative Test of Net Wound Healing in Rabbit Lepus negricollis (negricollis) between Provision of $10 \%$ Povidone iodine Solution and Snail Mucus (Achatina fulica). Thesis, Medical Faculty of Tandulako University; 2009.

13. Zulaechah S. The Difference between Sayat Speed Wound Healing using Snail Slime (Achatina fulica) with 10\% Povidone Iodine in Wound Care on Mice (Mus musculus). Yogyakarta: Thesis, Faculty of Medicine and Health Sciences, Muhammadiyah University; 2010.

14. Harti AS, Sulisetyawati SD, Murharyati A, Oktariani M, Wijayanti IB. The effectiveness of snail slime and chitosan in wound healing. Int $\mathrm{J}$ Pharm Med Biol Sci 2016;5:76-80.

15. Kozier B, Taylor CR. Fundamental of Nursing. $7^{\text {th }}$ ed. New Jersey: Education Inc.; 2004. p. 856-62.

16. Mandala AP, Agnes SH. Provision of $100 \%$ Snail Mucus (Achantina fulica) and 5\% Cream Preparation for Long-Term Healing Wound Healing (grade a) in vivo. Surakarta: Final Report, Departement of NURSING, Kusuma Husada Surakarta School of Health Science; 2014.

17. Johnson KE. Quick Review of Histology and Cell Biology. South Tangerang: Binarupa Publishing; 2011. 